\title{
Microbial growth modelling under variable temperature conditions
}

\author{
V. Popov ${ }^{1}$, R. Gospavic ${ }^{1}$, J. Kreyenschmidt ${ }^{2} \&$ S. Bruckner ${ }^{2}$ \\ ${ }^{1}$ Wessex Institute of Technology, Ashurst Lodge, Ashurst, UK \\ ${ }^{2}$ Institut für Tierwissenschaften, University of Bonn, Bonn, Germany
}

\begin{abstract}
The application of a Quantitative Microbial Risk Assessment (QMRA) in food supply chains requires accurate prediction of microbial growth under variable temperature conditions. A dynamical growth model under variable temperature conditions was developed using raw data for microbial growth of Pseudomonas spp. in poultry under aerobic conditions from a food supply chain in Germany. The primary model was developed using measurement data under a set of fixed temperatures and constant environmental conditions. The two primary models used for modelling the growth under constant temperature conditions were: Baranyi and modified Gompertz. As a secondary model the square root model was used for maximal growth rate in the Baranyi model and polynomial interpolation for the modified Gompertz model. In the case of the Baranyi model the main assumption, that the initial physiological state of the inoculum is constant and independent of the environmental parameters, is used. The generated dynamical models are validated by comparing the predictions obtained by both models with data measured under dynamical temperature conditions. Two temperature profiles were used for comparison and good agreement has been found between experimental data and model predictions.
\end{abstract}

Keywords: microbial growth, predictive microbiology, pseudomonas spp., poultry.

\section{Introduction}

The mathematical model for microbial growth is a necessary component in the efficient assessment of food contamination, shelf life and risk assessment in supply chains [1]. 
One of the most important components in quantitative microbial risk assessment (QMRA) systems is the development of a predictive microbial growth model which will be able to take into account the influence of the variation of environmental parameters on the microbial growth along the supply chain [2].

In recent times a considerable effort has been made in the development of mathematical models of microbial dynamics in food products and a number of mathematical models and expressions for predictive microbial growth in food have been developed [3].

In all cases the microbial growth under variable environmental conditions is described by first order kinetics i.e. by a single or by a system of ordinary differential equations of first order [4].

One of the most important environmental parameters, from the food safety and quality point of view, is temperature. Considering the temperature changes along the supply chain and the use of dynamical models which are able to take into account the influence of temperature variation on microbial growth is essential for risk assessment when considering food borne pathogens and/or for prediction of products' shelf life when considering spoilage microorganisms [5].

Basically there are two different ways to obtain a dynamical mathematical model for predictive microbial growth under variable temperature conditions. In the static experimental approach two steps are employed. In the first step the microbial growth data under constant environmental conditions is generated and this procedure is repeated for different sets of environmental parameters.

The obtained raw data is fitted with a so-called primary model i.e. with model curves that describe the growth of the microbial population with time. Some of the most common primary models are modified Gompertz, Baranyi and Roberts and Logistic models [6].

Once the model parameters under stationary environmental conditions are determined the second step is performed and the parameters' dependence on temperature is evaluated.

In this paper the microbial growth of Pseudomonas spp. in poultry under variable temperature conditions is considered. Primary and secondary models for temperature variations are developed and the obtained numerical results are validated under temperature variations through comparison with the measurements.

For primary models the Baranyi and Roberts and modified Gompertz models are used to fit the raw data for microbial growth under constant temperatures to obtain the primary model curves. In this way the model parameters for each selected temperature are obtained. In the next step the secondary model is developed where the functional dependence of the model parameters on temperature is achieved. As the temperature range was $2^{\circ} \mathrm{C}-20^{\circ} \mathrm{C}$ for the Baranyi and Roberts model the square root secondary model was used for maximal growth rate [7]. The maximal bacterial concentration, as well as the initial physiological state of the cells, is assumed to be temperature independent in the considered temperature range. For the Gompertz model the logarithmic transformation and polynomial interpolation is used as the secondary model. 
The obtained dynamical models were validated through a comparison of obtained numerical results and measurement of bacterial concentration under dynamical temperature conditions.

\section{Materials and methods}

\subsection{Experimental design}

Poultry filets, slaughtered $20 \mathrm{~h}$ previously, were purchased from a German wholesaler. Samples were transported in $15 \mathrm{~min}$ in a cooling box to the laboratory. The filets were sliced under sterile conditions into $100 \mathrm{~g}$ pieces, put in individual pouches and wrapped with low density polyethylene films. The pieces were stored under controlled temperature conditions in high precision low temperature incubators (Sanyo MIR 153) at 2, 4, 10, 15 and $20^{\circ} \mathrm{C}$. Two different dynamical storage experiments were conducted. In the first non-isothermal experiment the following cycle was used: $12 \mathrm{~h}$ at $10^{\circ} \mathrm{C}, 12 \mathrm{~h}$ at $2^{\circ} \mathrm{C}$. In the second cycle the temperature was varied in the following fashion: $10 \mathrm{~h}$ at $4{ }^{\circ} \mathrm{C}, 5 \mathrm{~h} 10^{\circ} \mathrm{C}$, $4 \mathrm{~h} 15^{\circ} \mathrm{C}$. During the experiments temperature was controlled every 5 minutes by data loggers (Testo 151) and samples from the filets were taken at appropriate time intervals. Every measurement was repeated at least 6 times. To characterize the spoilage process of poultry filets sensory and microbiological investigations were conducted.

\subsection{Sample preparation and microbiological analysis}

For microbiological analysis a representative product sample of $25 \mathrm{~g}$ was transferred to a Stomacher-bag and homogenized for $60 \mathrm{~s}$ in a Stomacher 400 (Kleinfeld Labortechnik) with $225 \mathrm{~g}$ chilled saline peptone diluent $(0.85 \% \mathrm{NaCl}$ with $0.1 \%$ petone). Further appropriate 10 -fold dilution of the homogenate was made with saline peptone diluent. For each dilution blank, two replicas were prepared. $0.1 \mathrm{ml}$ from each appropriate dilution step were spread on the surface of dried media into Petri dishes. Pseudomonas spp. were determined by using Pseudomonas agar base (Oxoid) plus CFC supplement (Oxoid). Petri dishes were aerobically incubated at $25^{\circ} \mathrm{C}$ for $48 \mathrm{~h}$.

\subsection{The primary model and mathematical modelling under dynamical temperature conditions}

The microbial growth under dynamical temperature conditions according to the Baranyi and Roberts model could be described by the following set of differential equations with appropriate initial conditions [3]

$$
\begin{gathered}
\frac{d q(t)}{d t}=v \cdot q(t) ; \quad q(0)=q_{0} \\
\frac{d N(t)}{d t}=\mu_{0} \cdot \alpha(t) \cdot\left(1-\left(\frac{N(t)}{N_{\max }}\right)^{m}\right) \cdot N(t) ; \quad \alpha(t)=\frac{q(t)}{1+q(t)} ; \quad N(0)=N_{0},
\end{gathered}
$$


where $q_{0}$ and $q(t)$ are the quantities that are related to the critical substance necessary for growth and characterize the physiological state of the culture in the moment of inoculation and later time, respectively. The temperature-dependent specific growth rate, expressed in $[1 / \mathrm{h}]$, is denoted by $\mu_{0} . N_{0}, N_{\max }$ and $N(t)$ are initial, maximal and actual cell concentration, respectively, expressed in $[\mathrm{cfu} / \mathrm{g}]$, $m$ is a shape parameter for which $m=1$ was assumed. The adjustment function, which takes into account the lag phase during which the population adapts to the new environment, is denoted by $\alpha(t)$. The relative growth rate $v$ relates to critical substance and determines the quickness of the transition from the lag phase to the exponential phase. It is assumed that the specific growth rate for quantity $q$ is equal to the relative growth rate of the colonies (i.e. $v=\mu_{0}$ ). The "logistic" part in equation (2) limits the population growth to the value $N_{\max }$. The growth rate is mainly determined by the environmental conditions.

The main assumptions for the dynamical temperature conditions are that the specific growth rate is changing instantaneously with temperature and that parameters $N_{\max }$ and $q_{0}$ are temperature independent. If the temperature variation in time is described by the temperature profile $T(t)$, the above system of equations could be solved by integration in the following way:

$$
\begin{gathered}
q(t)=q_{0} \cdot \exp \left(\int_{0}^{t} \mu_{0}\left(T\left(t_{1}\right)\right) d t_{1}\right) \\
y(t)=y_{0}+A(t)-\ln \left(1+\frac{\exp (A(t))-1}{\exp \left(y_{\max }-y_{0}\right)}\right) ; \quad A(t)=\int_{0}^{t} \mu_{0}\left(T\left(t_{1}\right)\right) \frac{q\left(t_{1}\right)}{1+q\left(t_{1}\right)} \cdot d t_{1} ; \\
y(t)=\ln (N(t)) ; \quad y_{0}=\ln \left(N_{0}\right) ; \quad y_{\max }=\ln \left(N_{\max }\right) .
\end{gathered}
$$

The quantity $y(t)$ in the equations mentioned above is the natural logarithm of the cell concentration $N(t)$. Function $A(t)$ plays the roll of the time delay, which is related to the transition from the adjustment to the growth phase, i.e. the lag time under dynamical conditions.

Instead of using parameter $q_{0}$, the quantity $h=\ln \left(1+1 / q_{0}\right)$ is more stable in practical calculations. For constant environmental conditions the growth curve for the Baranyi and Roberts model could be expressed by the following equation:

$$
y(t)=y_{\text {max }}+\ln \left(\frac{1-\exp (-h)+\exp \left(\mu_{0} t-h\right)}{\exp \left(y_{\max }-y_{0}\right)+\exp \left(\mu_{0} \cdot t-h\right)-\exp (-h)}\right) ; \quad h=\ln \left(\frac{1+q_{0}}{q_{0}}\right) .
$$

The maximal specific growth rate could be defined as actual specific growth rate at the flexion point of the growth curve. The natural logarithm of the cell concentration at the flexion point is given by the following equation:

$$
y_{\text {flexion }}=y\left(t_{\text {flexion }}\right)=\ln \left(N_{\max }\right)-\frac{1}{2} \ln \left(\frac{N_{\max }}{N_{0}}+q_{0} \cdot\left(\frac{N_{\max }}{N_{0}}-1\right)\right) .
$$

Here it is important to emphasize that $\mu_{0}$ is a model parameter in the Baranyi and Roberts model with the dimension of the specific growth rate and $\mu_{\max }$ is the maximal specific growth rate i.e. actual specific growth rate at the flexion point of the growth curve. 
The modified Gompertz primary model for microbial growth could be expressed by the following equation [8]:

$$
y(t)=A+C \cdot \exp (-\exp (-B \cdot(t-M))),
$$

where $A, C, B$ and $M$ are model parameters and $y$ has the same meaning as in (4), i.e., natural logarithm of the cell concentration.

The parameter $M$ is the flexion point of the sigmodial curve given by (10), $A+C$ is the natural logarithm of the maximal concentration $y_{\max }$, and parameter $A$ is asymptotic value for $y(t)$ if the time is taken in minus infinity and does not have any biological meaning.

The maximal growth rate and the lag phase for the modified Gompertz model could be expressed by the following expressions [9]:

$$
\begin{aligned}
& \mu_{\max }=\frac{B \cdot C}{e} ; \quad \lambda=M-\frac{1}{B}(1-\exp (1-\exp (B \cdot M))) ; \\
& \exp (B \cdot M)>>1 \Rightarrow \lambda \approx M-\frac{1}{B} .
\end{aligned}
$$

In the case of dynamical temperature conditions first order kinetics should be used and growth of the bacterial populations could be described by the following first order differential equations with corresponding initial conditions:

$$
\frac{d y(t)}{d t}=B \cdot(y-A) \cdot \ln \left(\frac{C}{y-A}\right) ; \quad y(0)=A+C \exp (-\exp (-B \cdot M)) .
$$

The model parameter $M$ is included only in the initial conditions.

If we assume that parameters $A$ and $C$ are temperature independent and that only parameter $B$ is a function of the temperature the solutions could be expressed by the following integral equation:

$$
y(t)=A+\left(y_{\max }-A\right) \cdot \exp \left(-\exp \left(-\int_{0}^{t} B\left(T\left(t_{1}\right)\right) \cdot d t_{1}+C_{0}\right)\right), \quad C_{0}=B(T(0)) \cdot M .
$$

\subsection{Secondary model}

To obtain the temperature dependence of the specific growth rate $\mu_{0}$, measurements for a set of constant temperature conditions are performed. As the temperature range for dynamical conditions was $2{ }^{\circ} \mathrm{C}-20^{\circ} \mathrm{C}$ and thermal deactivation was not considered, the simplified version of the Ratkowsky model, namely square root model, was used as a secondary model for specific growth rate for the Baranyi and Roberts model. Accordingly, the specific growth rate as a function of temperature could be expressed by the following equation [10]:

$$
\sqrt{\mu_{\max }}=b \cdot\left(T-T_{\min }\right) \text {, }
$$

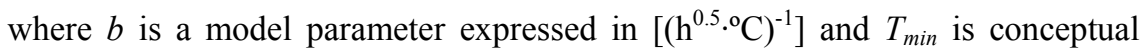
minimal temperature for microbial growth. Both parameters are obtained by fitting data for specific growth under different constant temperatures. The above model is valid only for the temperature range $\left[T_{\min }, T_{o p t}\right]$, where $T_{o p t}$ is the optimal temperature for microbial growth. 
For the modified Gompertz model the natural logarithm of the parameter $B$ is modelled as a second order polynomial function of temperature as follows [9]:

$$
\ln (B)=p_{0}+p_{1} \cdot T+p_{2} \cdot T^{2},
$$

where $p_{0}$ to $p_{2}$ are model parameters that are also determined by fitting of the model curves to the measured data.

\section{Results and discussion}

The static measurements were performed at five different temperatures: $2,4,10$, 15 and $20^{\circ} \mathrm{C}$. Obtained measurement data were converted in $\ln (\mathrm{cfu} / \mathrm{g})$ and were fitted to the growth curves for the primary model. In the case of Baranyi and Roberts in the first step model the four model parameters, namely: $y_{0}, y_{\max }, \mu_{\max }$, and $q_{0}$, were obtained by nonlinear regression using the software ORIGIN 7.5. As suggested by various authors [7], the parameter $h$ obtained for different temperatures is averaged and mean values $\langle h\rangle$ were used in the next step. This follows from the assumption that if the measurement procedures are standardised then initial physiological state of the colony, i.e. quantity $h$ should be the same for different temperatures during the static measurements.

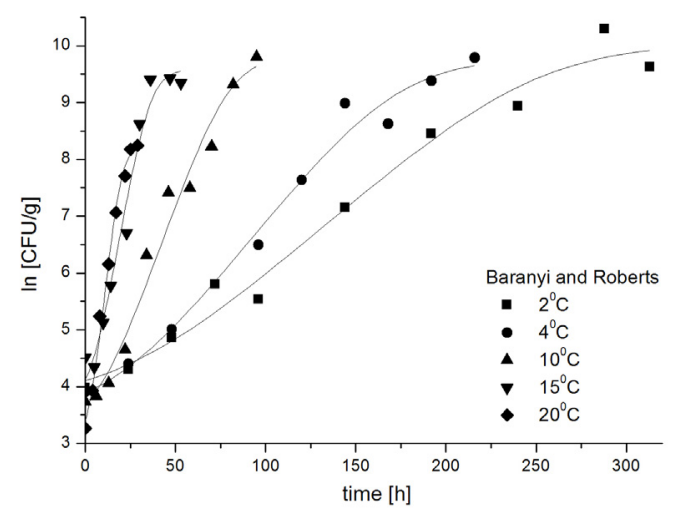

Figure 1: $\quad$ Raw data and fitted growth curves for Baranyi and Roberts model for five different temperatures $(<h>=1.0442)$.

In the second step the three remaining model parameters are recalculated with fixed value of the quantity $\langle h\rangle$ from the first step. The specific growth rate obtained in this way was used in the secondary model.

In Figures 1 and 2 the fitted growth curves at constant temperatures for Baranyi and Roberts and modified Gompertz primary models are shown respectively.

In Figures 3 and 4 the growth curves obtained using Baranyi and Roberts and modified Gompertz model for Pseudomonas spp. in poultry under dynamical temperature conditions are shown for two different temperature profiles. 


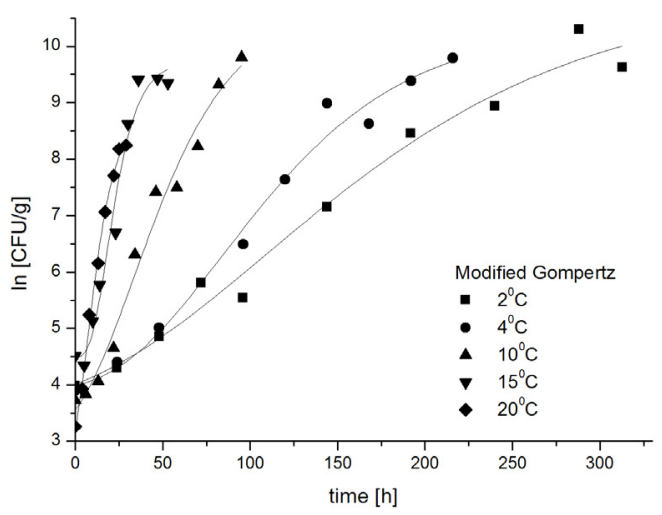

Figure 2: $\quad$ Raw data and fitted growth curves for modified Gompertz model for five different temperatures.

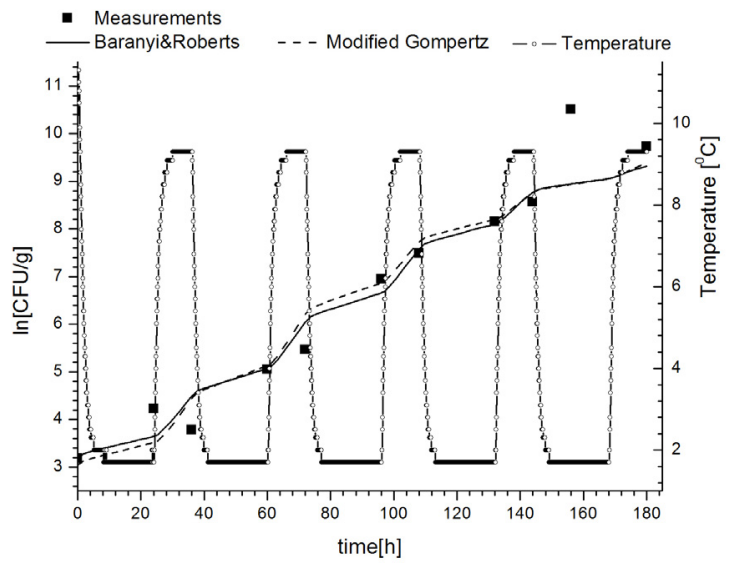

Figure 3: The growth curves for the Pseudomonas spp in poultry under dynamical temperature conditions (temperature profile 1) obtained using the Baranyi and Roberts and the modified Gompertz models compared with measurement results.

For the Baranyi and Roberts model the free parameter was the initial physiological state of the culture, namely $q_{0}$, and in the case of the modified Gompertz model the parameter $A$ was the free parameter. In both cases the free parameter was obtained by minimizing the MSE.

The obtained results are validated by comparison with measurements. The experimental measurements are repeated six times under the same temperature profiles and average values are used for comparison with model predictions. 


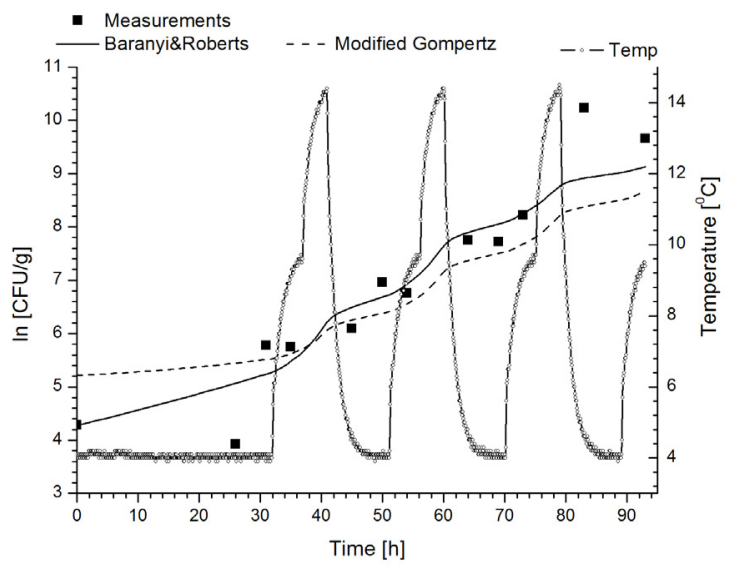

Figure 4: The growth curves for the Pseudomonas spp in poultry under dynamical temperature conditions (temperature profile 2) obtained using the Baranyi and Roberts and the modified Gompertz models compared with measurement results.

Table 1: $\quad$ The estimated optimal values for the model parameter $h$ and $A$ in the Baranyi and Roberts and modified Gompertz models, respectively, for two temperature profiles and relevant statistics for the validation of the models through comparison with measured data.

\begin{tabular}{|c|c|c|c|c|c|}
\hline \multicolumn{6}{|c|}{ Temperature profile 1} \\
\hline \multicolumn{3}{|c|}{ Baranyi and Roberts } & \multicolumn{3}{|c|}{ Modified Gompertz } \\
\hline $\mathrm{h}$ & MSE & $\begin{array}{c}\text { pseudo } \\
\text { R2 }\end{array}$ & A & MSE & pseudo R2 \\
\hline 0.5573 & 0.62547 & 0.936 & 2.5314 & 0.6478 & 0.9313 \\
\hline \multicolumn{6}{|c|}{ Temperature profile 2} \\
\hline 0.4106 & 0.60911 & 0.8999 & 5.1285 & 0.88317 & 0.7895 \\
\hline
\end{tabular}

The obtained results for variable parameters $h$ and $A$ for Baranyi and Roberts and modified Gompertz model, respectively, and the relevant statistics for the validation of the mathematical models used for the growth of Pseudomonas spp. in the poultry for two temperature profiles 1 and 2 are presented in Table 1.

\section{Conclusions}

Baranyi and Roberts and Gompertz models have been applied for the prediction of growth of Pseudomonas spp. in poultry under variable temperature conditions. The secondary models used are the Ratkowsky model (square root model) for the Baranyi and Roberts and the polynomial of second order for the Gompertz model. 
The results show that the confidence band for the Gompertz model is considerably larger than for the square root one used with the Baranyi and Roberts model. Though the comparison of the predictions of the two models with the experimental data under dynamical temperature conditions show good agreement in the case of growth of Pseudomonas spp. in poultry, the Baranyi and Roberts model is in better agreement than the modified Gompertz model, especially for the second temperature profile shown in Figure 11.

\section{Acknowledgements}

The present study was supported by the CHILL-ON project, contract number: FP6-016333-2, as part of the Sixth Framework Programme, Priority 5, Food Quality and Safety, and also by the federal state North Rhine Westphalia (USL PN 33108, USL PN 33110).

\section{References}

[1] Olafsdottir, G., Lauzon, H.L., Martinsdottir, E., Kristbergsson, K., Influence of storage temperature on microbial spoilage characteristics of haddock fillets (Melanogrammus aeglefinus) evaluated by multivariate quality prediction. International Journal of Food Microbiology. 111, 112125.

[2] Nauta, M., Van der Fels-Klerx, I., Havelaar, A., 2005. A PoultryProcessing model for quantitative microbial risk assessment. Risk Analysis. 25, 85-98.

[3] Baranyi, J., Roberts, T.A., 1995. Mathematics of predictive food microbiology. International Journal of Food Microbiology. 26, 199-218.

[4] Poschet, F., Vereecken, K.M., Geeraerd, A.H., Nicolaï, B.M., Van Impe, J.F., 2005. Analysis of a novel class of predictive microbial growth models and application to coculture growth. International Journal of Food Microbiology. 100, 107-124.

[5] Giannakourou, M.C., Koutsoumanis, K., Nychas, G.J.E., Taoukis, P.S., 2005. Field evaluation of the application of time temperature integrators for monitoring fish quality in the chill chain. International Journal of Food Microbiology. 102, 323-336.

[6] Juneja, V.K., Huang, L., Thippareddi, H.H., 2006. Predictive model for growth of Clostridium perfringens in cooked cured pork. International Journal of Food Microbiology. 110, 85-92.

[7] Amézquita, A., Weller, C.L., Wang, L., Thippareddi, H., Burson, D.E., 2005. Development of an integrated model for heat transfer and dynamic growth of Clostridium perfringens during the cooling of cooked boneless ham. International Journal of Food Microbiology. 101, 123-144.

[8] Juneja, V.K., Melendres, M.V., Huang, L., Gumudavelli, V., Subbiah, J., Thippareddi, H., 2007. Modeling the effect of temperature on growth of Salmonella in chicken. Food Microbiology. 24, 328-335. 
[9] Gibson, M.A., Bratchell, N., Roberts, T.A., 1988. Predicting microbial growth: growth responses of salmonellae in laboratory medium as affected by $\mathrm{pH}$, sodium chloride and storage temperature. International Journal of Food Microbiology. 6, 155-178.

[10] Ratkowsky, D.A., Lowry, R.K., McMeekin, T.A., Stokes, A.N., Chandler, R.E., 1983. Model for bacterial culture growth rate through the entire biokinetic temperature range. Journal of Bacteriology. 154, 1222-1226. 